\title{
Radiotherapie bei NSCLC: Höher dosiert, aber seltener
}

\section{Profitieren Patienten mit inopera- blem lokal fortgeschrittenem nicht- kleinzelleigem der Lungekrebs (NSCLC) von einer hypofraktionier- ten Bestrahlung?}

tandardtherapie bei Patienten mit in$\checkmark$ operablem lokal fortgeschrittenem NSCLC ist die hochdosierte Bestrahlung mit 60-70 Gy in 30-35 Fraktionen in Kombination mit einer platinbasierten Chemotherapie - das Überleben beträgt median 16-17 Monate. Von einer hypofraktionierten Strahlentherapie erhofft man sich eine bessere lokale Kontrolle, da ein größerer biologischer Effekt bei kürzerer Behandlungsdauer erzielt wird.

In der vorliegenden Studie wurde das bei 30 Patienten untersucht $(27 \%$ im Sta- dium IIIA, 50\% im Stadium IIIB und $23 \%$ im Stadium IV). Alle unterzogen sich einer hypofraktionierten und bildgebungsgeführten Strahlentherapie (60 Gy in 20 Fraktionen). Alle Patienten erhielten die vollen 60 Gy. Nach median 13 Monaten betrug die Gesamtansprechrate $83 \%$, mit drei (10\%) vollständigen und 22 (73\%) partiellen Remissionen. Zu lokaler Progression bzw. Rezidiven kam es bei $37 \%$ der Patienten, zu Fernmetastasen bei $57 \%$. Das mediane Gesamtüberleben betrug zwölf Monate, nach zwei Jahren lag die Überlebensrate bei 38,1\%.

Die Therapie wurde gut vertragen. Die häufigsten Toxizitäten waren Strahlenpneumonitis (Grad 1-2: 20\%, Grad 3: $7 \%$ ) und Ösophagitis (Grad 1-2: $40 \%$, Grad 3: 3\%). 17\% der Patienten hatten hämatologische Toxizitäten der Grade 1-2 und $3 \%$ vom Grad 3. Darüber hinaus entwickelten $33 \%$ der Patienten späte Toxizitäten der Grade 1-2 und 7\% vom Grad 3, vor allem Ösophagitis und Pneumonitis.

Fazit: Die Toxizität der hypofraktionierten Strahlentherapie war akzeptabel. Sie lässt auf bessere lokale Tumorkontrolle und längeres Überleben bei fortgeschrittenem NSCLC hoffen, muss aber noch randomisiert gegen die konventionelle Therapie getestet werden. Judith Neumaier

Osti MF et al. Image guided hypofractionated 3-dimensional radiation therapy in patients with inoperable advanced stage non-small cell lung cancer. Int J Radiat Oncol Biol Phys. 2013;85(3):e157-63.

\section{Tyrosinkinasehemmer zusätzlich zur Bestrahlung?}

20-40\% der Patienten mit nichtkleinzelligem Lungenkarzinom (NSCLC) entwickeln Hirnmetastasen. Nach Ganzhirnbestrahlung leben sie im Median noch drei bis sechs Monate. Kann ein EGFR-Inhibitor ihr Leben verlängern?

E ne Bestrahlung steigert die Expression des epidermalen Wachstumsfaktorrezeptors (EGFR) in Tumorzellen, was vermutlich zur Resistenz führt. $\mathrm{Ob}$ dieser Effekt durch eine EGFR-Blockade reduziert werden kann, wurde in einer Phase-II-Studie mit dem EGFR-Inhibitor Erlotinib und Ganzhirnbestrahlung an 40 Patienten mit NSCLC und Hirnmetastasen untersucht. Die Patienten Tagen pro Woche, bis zu $35 \mathrm{~Gy}$ ). waren median59 Jahre alt, die meisten hatten vier oder mehr Hirnmetastasen. Erlotinib wurde sechs Tage lang einmal täglich in einer Dosis von $150 \mathrm{mg}$ verabreicht, anschließend zusammen mit der Ganzhirnbestrahlung (2,5 Gy an fünf

Alle 40 Patienten vertrugen die Behandlung gut. Es war kein Anstieg der Neurotoxizität zu beobachten und keine

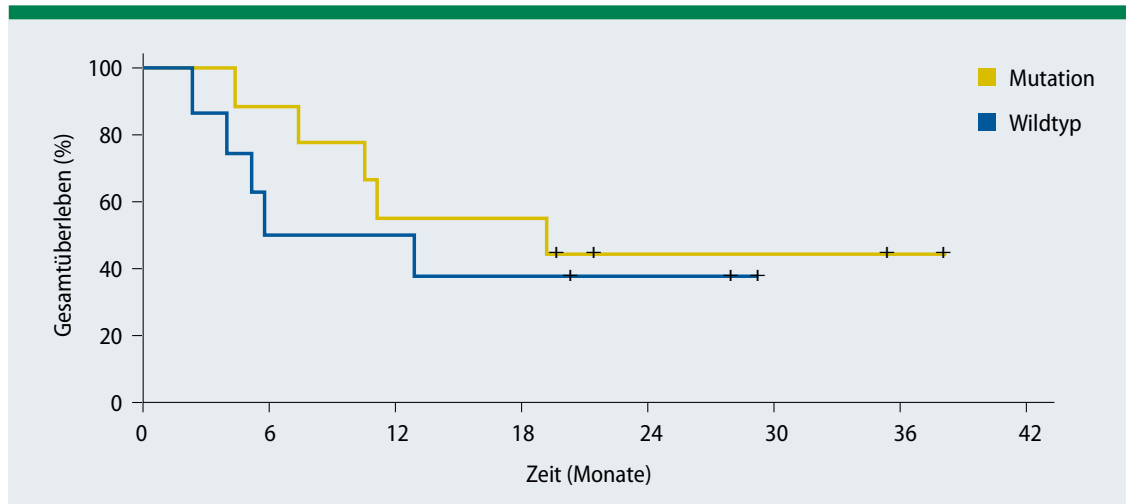

Abb.: Bei einem Wildtyp des EGFR-Rezeptors ist das Gesamtüberleben schlechter.
Toxizitäten vom Grad 4 oder 5. Die häufigste Nebenwirkung war Hautausschlag (67,5\% der Patienten), bei zwei Patienten war eine Dosisreduktion erforderlich, $70 \%$ erhielten für mindestens einen weiteren Monat Erlotinib in Monotherapie.

Das Gesamtansprechen betrug $86 \%$ ( $\mathrm{n}=36$ ), das mediane Überleben 11,8 Monate, das 1-Jahres-Überleben $50 \%$, das 2-Jahres-Überleben $24 \%$.

Von 17 Patienten mit bekanntem EGFR-Status hatten neun Mutationen, eine unerwartet hohe Rate bei diesen unselektierten Patienten. Hautausschlag trat bei ihnen häufiger auf. Auch beim medianen Gesamtüberleben gab es einen signifikanten Unterschied zwischen Patienten mit Wildtyp- und mutiertem EGFR: 9,3 versus 19,1 Monate.

Fazit: Die Kombination aus Erlotinib und Ganzhirnbestrahlung wurde gut vertragen. Die objektive Ansprechrate war hoch, die Überlebensraten besser als in früheren Studien, wobei Patienten mit EGFR-Mutationen am stärksten profitierten. Eine Überprüfung in größeren Kollektiven ist nötig. Judith Neumaier

Welsh JW et al. Phase II trial of erlotinib plus concurrent whole-brain radiation therapy for patients with brain metastases from non-small-cell lung cancer. J Clin Oncol. 2013;31(7):895-902. 